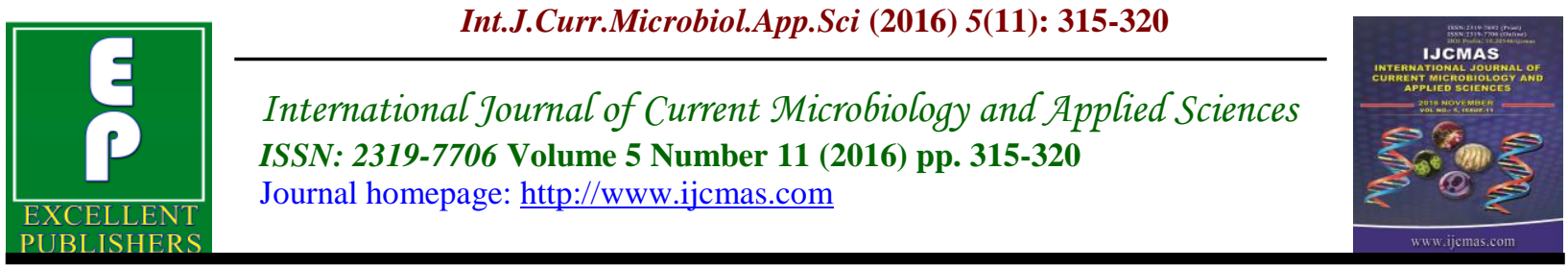

Original Research Article

http://dx.doi.org/10.20546/ijcmas.2016.511.034

\title{
Phenotypic Detection of Carbapenemase Producing Gram Negative Bacteria by Modified Hodge Test
}

\author{
S. Singh*, S.A. Samant, M. Bansal, A. Talukdar and D. Arif \\ Department of Microbiology, MGM Medical College and Hospital, \\ Kamothe, Navi Mumbai, India \\ *Corresponding author
}

\begin{tabular}{|c|c|}
\hline & $\mathbf{R} A \mathbf{C} \mathbf{T}$ \\
\hline s. & \multirow{3}{*}{$\begin{array}{l}\text { The acquisition of metallo- } \beta \text {-lactamases (Carbapenemase) by Gram Negative } \\
\text { Bacteria has recently emerged as one of the most worrisome resistance } \\
\text { mechanisms, a virtue by which they can hydrolyze all beta-lactam antibiotics } \\
\text { including penicillins, cephalosporins and carbapenems. This study was undertaken } \\
\text { for phenotypic detection of Carbapenamase in Gram Negative Bacteria by } \\
\text { Modified Hodge Test. A total of } 200 \text { isolates of Gram Negative Bacteria recovered } \\
\text { from various clinical specimens. They were subjected to antimicrobial sensitivity } \\
\text { testing and Modified Hodge Test. It was found that } 46 \text { isolates of Gram Negative } \\
\text { Bacteria showed Carbapenamase production by Modified Hodge Test out of which } \\
29 \text { isolates were resistant of Carbapenems by disc diffusion. However the } \\
\text { remaining } 17 \text { isolates could be potential Carbapenamase producers and may lead to } \\
\text { treatment failure. Hence it is necessary to carry out Modified Hodge Test for Gram } \\
\text { Negative Bacteria. }\end{array}$} \\
\hline & \\
\hline & \\
\hline
\end{tabular}

\section{Introduction}

Gram-negative bacteria have become increasingly resistant to available antibiotic drugs. Some strains are now resistant most of the available treatments resulting in increased illness and death from bacterial infections, and contributing to escalating healthcare costs. Examples of Gramnegative bacteria that have demonstrated drug resistance include: Escherichia coli, which causes the majority of urinary tract infections, Acinetobacter baumannii, which causes disease mainly in healthcare settings, Pseudomonas aeruginosa, which causes bloodstream infections and pneumonia in hospitalized patients and is a common cause of pneumonia in patients with cystic fibrosis, Klebsiella pneumoniae, which causes many types of healthcare-associated infections, including pneumonia, urinary tract infections, and bloodstream infections (Antimicrobial drug resistance, 2001; Anton et al., 2010; CDC guideline-Department of health \&human; Cheol-In Kang, 2013).

Multidrug resistant Gram-negative bacteria are an increasing therapeutic challenge, the major cause of which is beta lactamase production. Carbapenem-resistant Enterobacteriaceae (CRE) are usually resistant to all $\beta$-lactam agents as well as most other classes of antimicrobial agents. The treatment options for patients infected with CRE are very limited. Carbapenem 
resistance in Enterobacteriaceae occurs when an isolate acquires a carbapenemase or when an isolate produces an extendedspectrum cephalosporinase, such as an AmpC-type $\beta$-lactamase, in combination with porin loss. Various methods like, EDTA disk synergy (EDS) test, MBL E-test, EDTA-based microbiological assay are used for detection of MBLs (Noyal et al., 2009). For isolates that test susceptible to a Carbapenem but demonstrate reduced susceptibility either by disk diffusion or MIC testing, the Modified Hodge Test (MHT), is recommended. CLSI also recommends use of Modified Hodge Test for detection of Carbapenamase. Hence this study was taken up to isolate and identify the Gram negative bacilli from various clinical samples and to determine their Carbapenemase activity by Modified Hodge test.

\section{Materials and Methods}

This study was conducted over a period of one year (December 2013 to December 2014), in the Department of Microbiology, MGM Medical College \& Hospital, Kamothe, Navi Mumbai. A total of 200 isolates of Gram Negative Bacteriarecovered from clinical specimens likeurine, pus, blood, body fluids, sputum, Central line tip, Catheter tip etcwere included in the study.
They were identified using standard microbiological procedures (Koneman, 2002). They were also subjected to antimicrobial susceptible test by Kirby baur disc diffusion method (Bauer et al., 1966).

All the isolates were tested for Carbapenemase production by Modified Hodge Test

\section{Modified Hodge Test}

\section{Procedure}

0.5 McFarland dilution of the E.coli ATCC 25922 in $5 \mathrm{ml}$ of Broth or saline was prepared.A lawn of the 1:10 dilution of E.coli ATCC 25922 was streaked on a Mueller Hinton agar plate and allowed to dry for 3-5 minutes. A $10 \mu \mathrm{g}$ Imipenem, disc was placed in the center of the plate. In a straight line, test organism from the edge of the disc to the edge of the plate was streaked.

Plates were incubated overnight at $35^{\circ} \mathrm{C} \pm$ $2{ }^{\circ} \mathrm{C}$ for 16-24 hours. After 16-24 hours of incubation, the plates were examined for a clover leaf-type indentation at the intersection of the test organism and the E. coli 25922, within the zone of inhibition of the Carbapenem susceptibility disc.

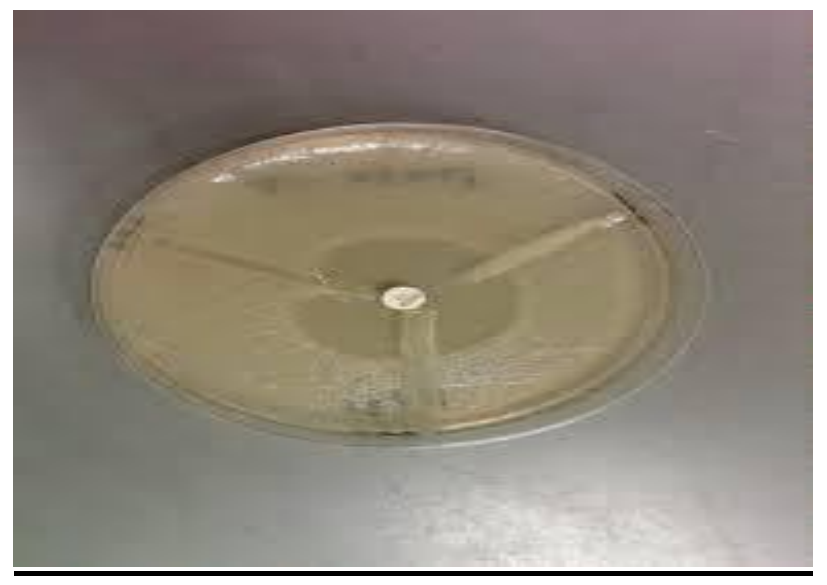

MHT Positive test has a clover leaf-like indentation of the E.coli 25922 growing 
along the test organism growth streak within the disc diffusion zone.

MHT Negative test has no growth of the E.coli 25922 along the test organism growth streak within the disc diffusion (CDCCenter of disease control).

\section{Result \&Discussion}

The cloverleaf technique, or Modified Hodge test (MHT), has been extensively used as a technique for detecting Carbapenemase activity. It is easily available in clinical microbiology routine settings and recommended by the CLSI for phenotypic detection of Carbapenemase.

MHT is based on the inactivation of a Carbapenem by Carbapenemase-producing strains that enable a Carbapenem-susceptible indicator strain to extend growth towards a Carbapenem-containing disk, along the streak of inoculum of the tested strain. In this study total 200 Gram negative pathogens were isolated from various clinical samples, which include urine, pus, sputum, blood, body fluids etc. figure1 shows spectrum of various Gram negative bacteria's in these cinical specimens.

In similar study done at Iran by A.Amjad.et.al (2011) reported 69\% of Carbapenemase producers among the 200 Gram negative bacteria by Modified Hodge test. In an Indian study done at K.V. Institute Kanchipuram by Balan. $\mathrm{K}$ et.al (2012) reported Carbapenemase production in about $22.5 \%$ out of 200 isolates by Modified Hodge test (Balan et al., 2013).

In another Indian study Noyal et al., (2009) from Pondicherry, conducted Modified Hodge test on Acinetobacter spp. and Pseudomonas spp. and reported $31 \%$ and
18\% Carbapenemase activity in Acinetobacter spp. and Pseudomonas spp. respectively. Figure 3 shows percentage of various isolates showing Carbapenemase activity. Maximum Carbapenemase activity was shown by Klebsiella (31\%) followed by Acinetobacter spp. (28\%) \& E.coli $(15.5 \%)$, Pseudomonas (15.2\%).

Amudhan et al., in 2011 isolated total 116 non-duplicate Carbapenem resistant Acinetobacter baumannii from various clinical samples and performed Modified Hodge test on them. A total of 113 Acinetobacter spp. isolates showed Carbapenemase activity (Amudhan et al., 2011).

Table 1 shows prevalence of Carbapenem resistance by Kirby Bauer's disc diffusion method. Out of 200 Gram negative bacteria isolated from various clinical specimens 66 strains were isolated from Urine out of which 26(39.4\%) strains were Carbapenem resistant. Out of 49 strains isolated from Pus $15(30.6 \%)$ strains were Carbapenem resistant. Out of 14 strains obtained from sputum 5(35.7\%) were Carbapenem resistance. Carbapenem resistance was observed in 3 out of 9 strains isolated from blood.

In our study 62 isolates were obtained from body fluids and patients with Endotracheal tip, Catheter tip, Central line tip. Out of 62 isolates 29(46.8\%) were resistant to Carbapenems. This can be because of the fact that these patients had a long stay in the hospital and were on prolonged antibiotic treatment.

Table 2 shows that out of 46 Modified Hodge test positive isolates only 29 isolates had exhibited resistance against Carbapenem by Disk diffusion method; while out of 46 
Table.1 Prevalence of carbapenem resistance amongst gram negative bacteria isolated from various clinical samples (Disk Diffusion Method)

\begin{tabular}{|l|l|l|l|l|l|l|}
\hline Clinical Sample & Urine & Pus & Sputum & Blood & Et tip & Body fluids \\
\hline Total (200) isolates & 66 & 49 & 14 & 9 & 41 & 11 \\
\hline Carbapenem Resistance & 26 & 15 & 05 & 03 & 24 & 05 \\
\hline Percentage\% & $39.4 \%$ & $30.6 \%$ & $35.7 \%$ & $33.3 \%$ & $58.5 \%$ & $45.4 \%$ \\
\hline
\end{tabular}

Table.2 Carbapenem Resistance among Modified Hodge Test Positive Isolates:

\begin{tabular}{|l|l|l|}
\hline Total No. of Isolates $(+)$ & Carbapenem $(\mathbf{S})$ & Carbapenem $(\mathbf{R})$ \\
\hline 46 & 17 & 29 \\
\hline
\end{tabular}

Fig.1 Spectrum of Gram negative Pathogens in various clinical specimens

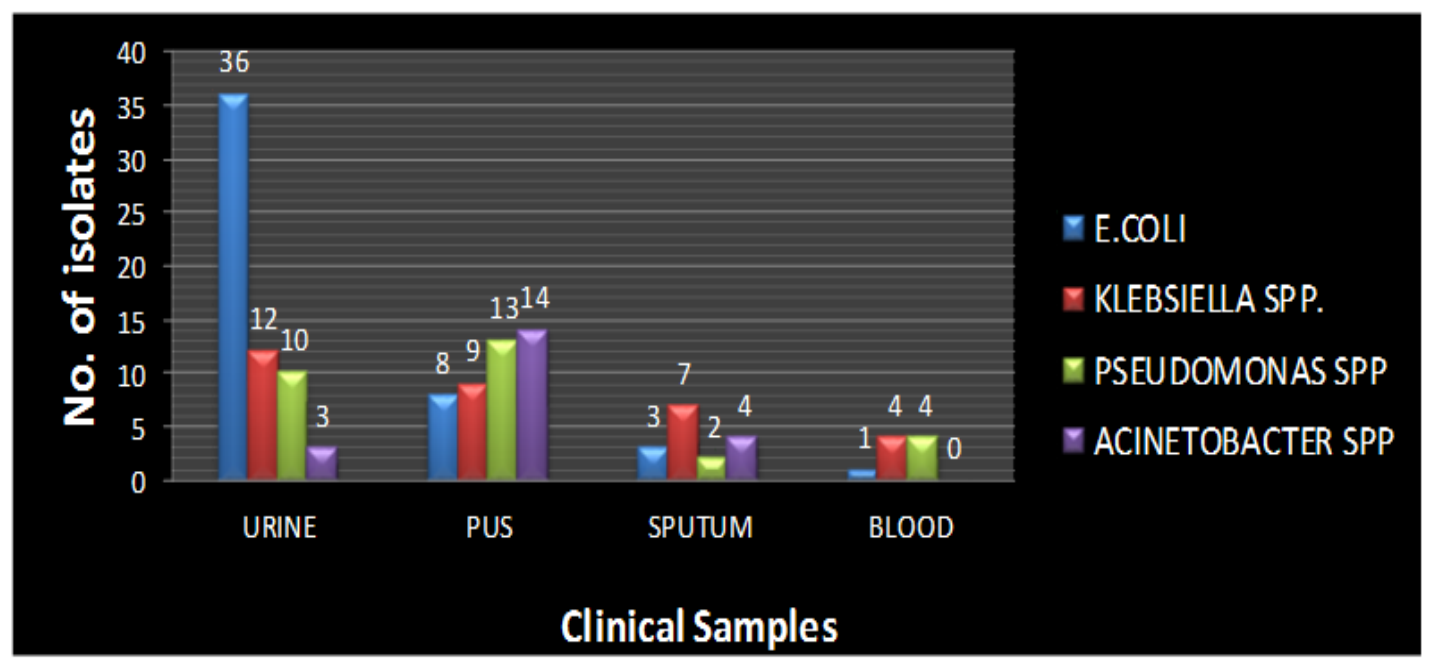

Fig.2 Shows prevalence of Carbapenemase activity in Gram negative isolates

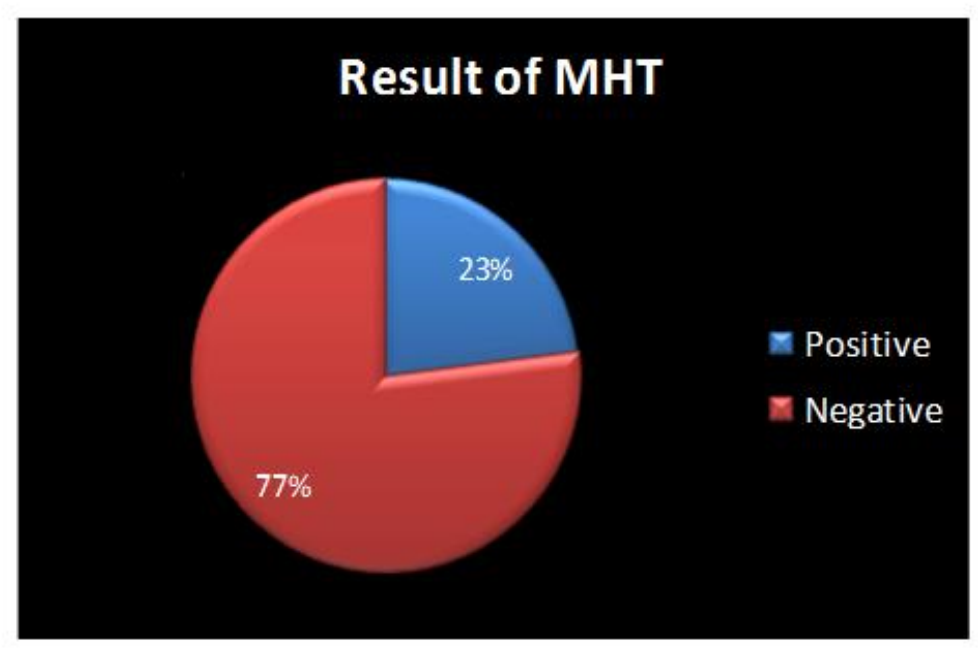


Fig.3 Carbapenemase activity detected in various Gram negative bacteria:

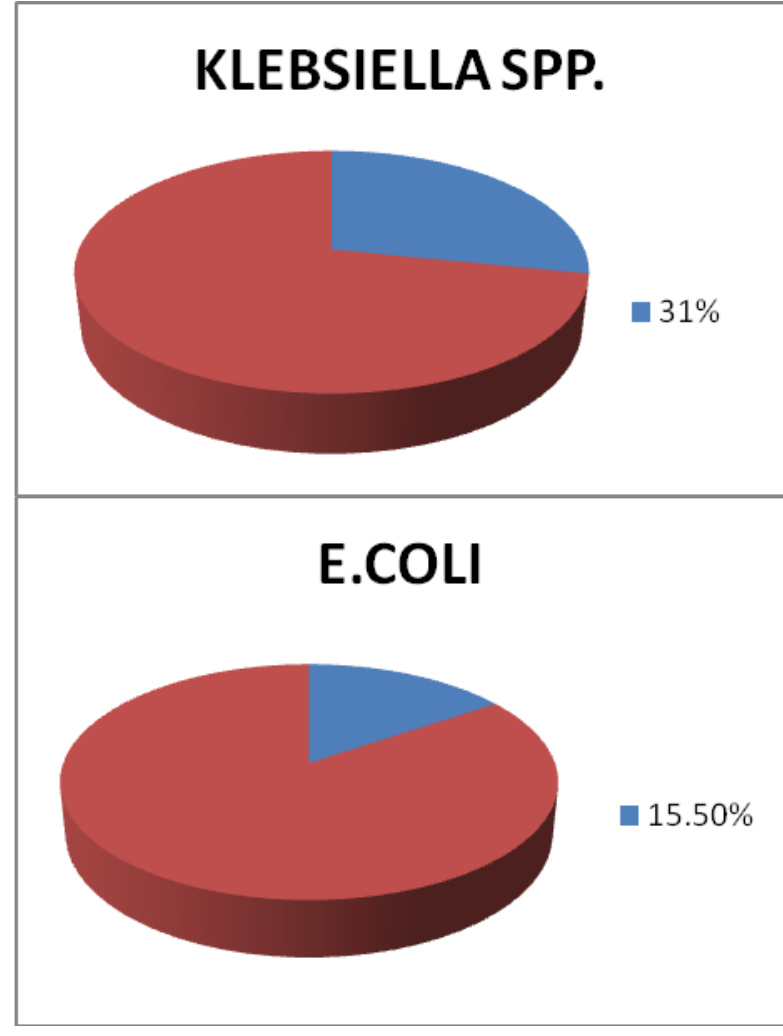

MHT positive $17(36 \%)$ isolates were sensitive to Carbapenem disk by Disk diffusion method. This indicates that even though the strains have not exhibited Carbapenem resistance by Disk Diffusion method they had the capacity to produce Carbapenemase which may give altered results in vivo.

In conclusion, out of 200 isolates 46 isolates (23\%) were found to be Carbapenemase producers when tested by Modified Hodge test. Amongst the various Gram negative bacteria maximum Carbapenemase activity was observed in Klebsiella spp. in which $31 \%$ isolates were Carbapenemase producers.

Maximum Carbapenemase producers were isolated from the patients with indwelling devices with long hospital stay and prolonged antibiotic treatment. These could be the resistant hospital strains. However

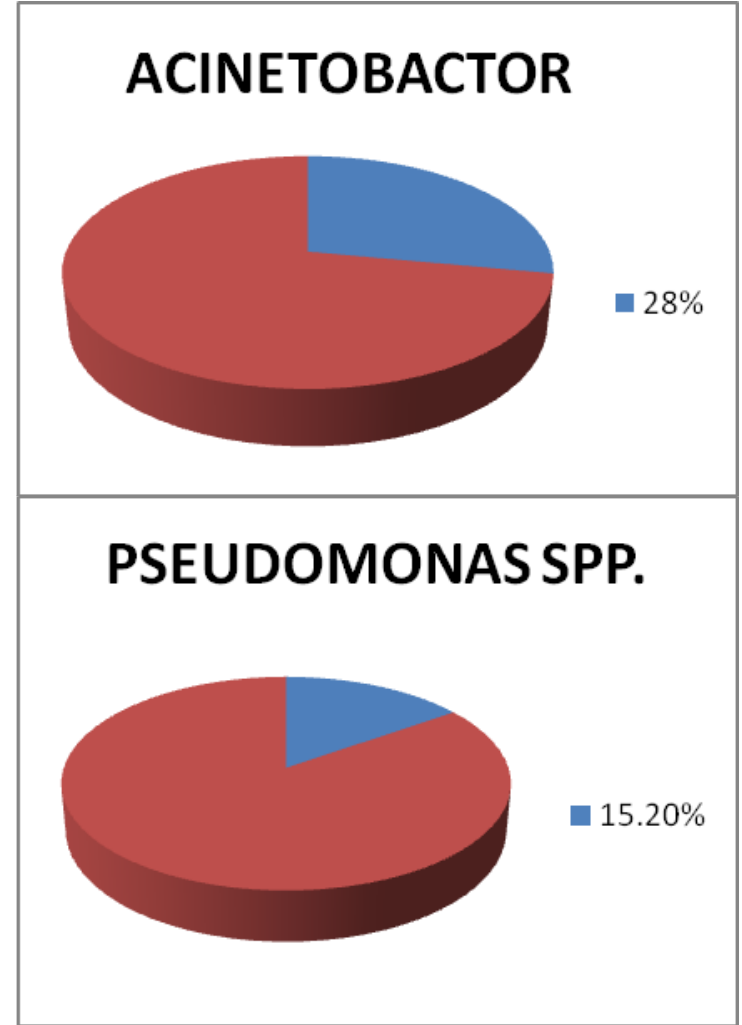

their association with hospital acquired infections could not be ascertained due to time constraints and unavailability of records.

Amongst the 46 Modified Hodge test positive Gram negative isolates 29 were resistant to Carbapenems and 17 were sensitive. These 17 isolates may be the isolates that are potential Carbapenems producers but have not expressed it in Disc Diffusion method. However this could be a matter of concern as these isolates may resist antibiotic treatment in vivo leading to treatment failure.

In conclusion, Modified Hodge test is an easy simple and reliable method to detect Carbapenemase producing Gram negative bacteria. There are a very high percentage of Gram negative bacteria showing Carbapenemase activity in out set up. It is 
imperative that all isolates showing intermediate or sensitive zone diameter on Disc Diffusion be tested for production of Carbapenemases by Modified Hodge test to avoid treatment failures and development of resistance due to unnecessary use of higher class antibiotics.

\section{References}

Amjad, A., Mirza, I.A., Abbasi, S.A., Farwa. U., Malik, Nzia, F. 2011. Prevalence of Carbapenemase in Gram negative isolates. Iran J. Microbiol., 3(4):189-193.

Amudhan, S.M et al. 2011. OXA betalactamase-mediated carbapenem resistance in Acinetobacter baumannii. Indian J. Med., 29(3): 269-274.

Antimicrobial drug resistance. 2001. National institute of allergy infectious diseases, vol 2: Pp157.

Anton, Y., Peleg, et al. 2010. Hospital Acquired Infections Due to GramNegative Bacteria. The New England J. Med., 362: 1804-1813.

Balan, K., Karpaga Vinayaga. 2013. Modified Hodge Test and Remodified Hodge Test for Carbapenemase Detection. Indian J. Appl. Res., 3 Issue: ISSN-2249-555X.

Bauer, A.W., Kirby, W.M.M., Sherris, J.C., Turch, M. 1966. Antibiotic Susceptibilty testing by a standardized single Disk method. AMJ by Clin. Patol., 36: 493496.

Calfee, D., Jenkins, S.G. 2000. Use of active surveillance cultures to detect asymptomatic colonization with carbapenem-resistant Klebsiella pneumoniae in intensive care unit patients. Infection Control Hospital Epidemiol., PMC (Public medical central) 129:966-968.

CDC guideline-Department of health \& human.

CDC-Center of disease control: Modified Hodge test.

Cheol-In Kang. 2013. Bloodstream Infections Caused by Antibiotic-Resistant GramNegative Bacilli. Risk Factors for Mortality and treatment outcome with special emphesis on Antimicrobial Therapy. American Society of Microbes, 57: Issue 12K. Lee. services, Center for Disease Control \& prevention.

Koneman's Colour Atlas and Textbook of Diagnostic Microbiology $6^{\text {th }}$ edition ; Lippincott Williams \& Wilkins;2002 Chapter 7 Part 1 Pp313-316.

Noya, M.J.C. 2009. Simple screening tests for detection of carbapenemases in clinical isolates of non fermentative Gramnegative bacteria. Indian J. Med. Res., 129: 707-712.

Noyal, M.J., Menezes, G.A., Harish, B.N., Sujatha, S., Parija, S.C. 2009. Simple screening tests for detection of carbapenemases in clinical isolates of nonfermentative Gram-negative bacteria. Indian J. Med. Res., 29: 707-712.

Wayne, P.A. 2009. Clinical and Laboratory Standards Institute/NCCLS. Performance Standards for Antimicrobial Susceptibility Testing. M100-S19. CLSI.

\section{How to cite this article:}

Singh, S., S.A. Samant, M. Bansal, A. Talukdar and Arif, D. 2016. Phenotypic Detection of Carbapenemase Producing Gram Negative Bacteria by Modified Hodge Test. Int.J.Curr.Microbiol.App.Sci. 5(11): 315-320. doi: http://dx.doi.org/10.20546/ijcmas.2016.511.034 